\title{
Prediction of Aircraft's Longitudinal Motion Based on Aerodynamic Coefficients and Derivatives by Surrogate Model Approach
}

\author{
Norazila Othman and Masahiro Kanazaki \\ Graduate School of System Design, Division of Aerospace Engineering, Tokyo Metropolitan University, Hino City 191-0065, Japan
}

Received: December 10, 2013 / Accepted: January 06, 2014 / Published: July 25, 2014.

\begin{abstract}
The accuracy of a flight simulation is highly dependent on the quality of the aerodynamic database and prediction accuracies of the aerodynamic coefficients and derivatives. A surrogate model is an approximation method that is used to predict unknown functions based on the sampling data obtained by the design of experiments. This model can also be used to predict aerodynamic coefficients/derivatives using several measured points. The objective of this paper is to develop an efficient digital flight simulation by solving the equation of motion to predict the aerodynamics data using a surrogate model. Accordingly, there is a need to construct and investigate aerodynamic databases and compare the accuracy of the surrogate model with the exact solution, and hence solve the equation of motion for the flight simulation analysis. In this study, sample datas for models are acquired from the USAF Stability and Control DATCOM, and a database is constructed for two input variables (the angle of attack and Mach number), along with two derivatives of the $X$-force axis and three derivatives for the $Z$-force axis and pitching moment. Furthermore, a comparison of the value predicted by the Kriging model and the exact solution shows that its flight analysis prediction ability makes it possible to use the surrogate model in future analyses.
\end{abstract}

Key words: Surrogate model, Kriging method, equation of motion, standard dynamics model.

\section{Introduction}

To predict the value of an unknown point, several types of surrogate models have been widely used in the engineering field, such as the Kriging model. In engineering design, high fidelity flight simulation is desirable for an unconventional airplane design such as a BWB (blended wing body), as well as for simulating a hazard, such as an emergency engine stop condition. However, the prediction of high fidelity aerodynamics requires a time-consuming flow solver. Many researchers have focused on predicting aerodynamic force and moment derivatives by using CFD (computational fluid dynamics) [1, 2].

Therefore, a prediction method is required when a function has to be evaluated via a time-consuming

Corresponding author: Norazila Othman, Ph.D. student, research fields: aerospace engineering and flight simulation. E-mail: ila_fathanah@yahoo.com. solver. Jeong et al. [3] showed that a surrogate model is suitable for efficient design optimization. Donald et al. [4] applied a Kriging-based genetic algorithm to aircraft design to reduce time-consuming aerodynamic evaluations. In addition, Shahrokhi et al. [5] successfully implemented a neural network method to evaluate the aerodynamics while considering the computation cost.

This paper focuses on the construction of a database of aerodynamic force derivatives and coefficients for efficient high fidelity flight simulation. The SDM (standard dynamics model), which has been used for various studies involving wind tunnel testing [6-9], is a simplified F-16 model with an engine intake, a strake, a delta wing, and vertical and horizontal tails and stabilizers [10]. The aerodynamic coefficients and derivatives are evaluated using the USAF Stability and Control DATCOM. DATCOM estimates the 
aerodynamic derivatives based on geometric details and flight conditions [1]. This paper is organized as follows: Section 2 presents an overview of the surrogate models, which are described using the general formulation of Kriging; Section 3 describes the equation of motion used for solving flight simulation problems based on the aerodynamic coefficients and derivatives predicted by the surrogate model; Section 4 gives the computational conditions used in the present study; Section 5 shows the results of the surrogate model, including the error estimation along with the characteristics of longitudinal motion; and the conclusions are explained in Section 6.

\section{Surrogate Models}

Surrogate models are methods that are used to approximate functions based on various sample points. In the following subsections, the details of each surrogate model considered here are described.

\subsection{Kriging Model}

The Kriging model expresses the value, $y\left(x_{i}\right)$ at an unknown design point $\left(x_{i}\right)$ using Eq. (1) [11-13]:

$$
y\left(x_{i}\right)=\mu+\varepsilon\left(x_{i}\right)(i=1,2, \ldots, m)
$$

where, $m$ is the number of design variables, $\mu$ is the constant global model and $\varepsilon$ is the local deviation from the global model. Using this model, the local deviation at an unknown point is expressed using stochastic processes, and some design points are calculated using the Gaussian random function as a correlation function to estimate the trend of the stochastic process.

Let $\hat{y}(x)$ be an approximation model, where ${ }^{\wedge}$ is the estimator. Thus, the Kriging approximation model can be described as follows:

$$
\hat{y}(x)=\hat{\beta}+r^{T}(x) R^{-1}(y-\hat{\beta} i)
$$

where, $\hat{\beta}$ is the estimated value of $\beta, R^{-1}$ is the inverse of the correlation matrix $R, r$ is the correlation vector, $y$ represents the observed data with $n_{s}$ sample data points, and $i$ is a unit vector which has $n_{s}$ components. The correlation matrix is defined as follows:

$$
\begin{gathered}
R\left(x^{j}, x^{k}\right)=\operatorname{Exp}\left[-\sum_{i=1}^{n} \theta_{i}\left|x_{i^{j}}-x_{i^{k}}\right|^{2}\right] \\
\left(j=1, \ldots, n_{s}, k=1, \ldots, n_{s}\right)
\end{gathered}
$$

where, $n$ is the number of design variables, and $\theta_{i}$ is the $i$-th parameter corresponding to the $i$-th design variable. The correlation vector is represented as follows:

$$
r(x)=\left[R\left(x, x^{(1)}\right),\left(x, x^{(2)}\right), \ldots .,\left(x, x^{\left(n_{s}\right)}\right)\right]^{T}
$$

The likelihood function, $L$, is defined as follows:

$$
L\left(u, \theta, \beta, \sigma^{2}\right)=\frac{\left(2 \pi \sigma^{2}\right)^{\frac{-n_{S}}{2}}}{\sqrt{|R|}} \times \operatorname{Exp}\left[-\frac{(y-\widehat{\beta} i)^{T} R^{-1}(y-\widehat{\beta} i)}{2 \pi \sigma^{2}}\right]
$$

By determining the partial derivatives of the logarithm of the likelihood function defined in Eq. (5) with respect to $\widehat{\beta}$ and $\sigma^{2}$, and letting them be equal to zero, the maximum likelihood estimators of $\widehat{\beta}$ and $\sigma^{2}$ are determined as follows:

$$
\begin{aligned}
& \hat{\beta}=\left(i^{T} R^{-1} i\right)^{-1} i^{T} R^{-1} y \\
& \widehat{\sigma^{2}}=\frac{(y-\widehat{\beta} i)^{T} R^{-1}(y-\widehat{\beta} i)}{n_{s}}
\end{aligned}
$$

\section{Equation of Motion for Flight Simulation}

\subsection{Simulation Target}

In this paper, the SDM is considered as an example [7-10]. The dimension of SDM is illustrated in Fig. 1. The initial moments of $I_{x x}, I_{y y}$ and $I_{y y}$ are $0.125 \mathrm{~kg} \cdot \mathrm{m}^{2}$, $0.539 \mathrm{~kg} \cdot \mathrm{m}^{2}$ and $0.616 \mathrm{~kg} \cdot \mathrm{m}^{2}$, respectively. The moment center is $35 \%$ of the MAC (mean aerodynamic chord).

\subsection{Aerodynamic Evaluation}

Generally, the aerodynamic coefficients and derivatives are evaluated for this model using the linear theory and semi-empirical expressions from the USAF Stability and Control DATCOM [1]. This makes it possible to evaluate the aerodynamic performance based on the empirical model. In this study, the angle of attack and Mach numbers are changed from $0^{\circ}$ to $30^{\circ}$ and 0.6 to 1.4 , respectively. 


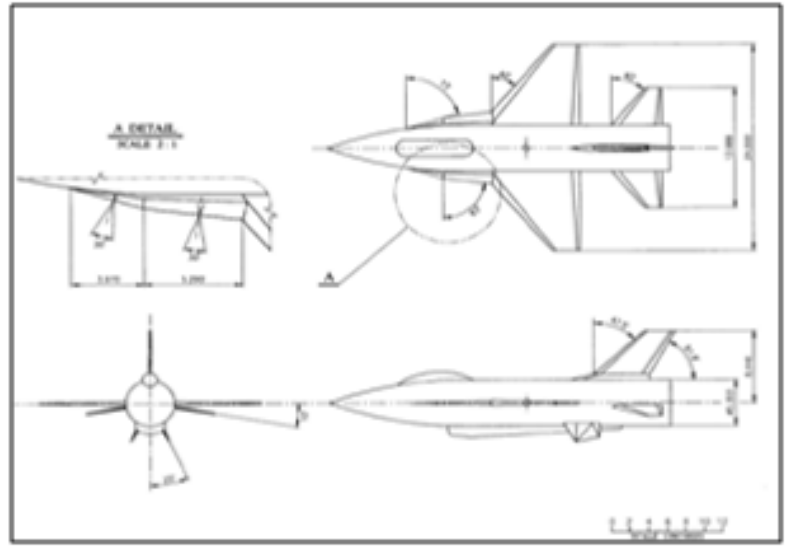

Fig. 1 Three-dimensional view of SDM [7-10].

\subsection{Equation of Motion for Flight Motion}

\subsubsection{Equations for Longitudinal Motion}

In this paper, the present flight simulation is applied to the longitudinal motion. The $X$ - and $Z$-force derivatives and pitching moment derivatives are involved in the calculation of the longitudinal motion. The general second-order differential equation for the flight simulation is expressed as Eq. (8):

$$
\frac{\mathrm{d}^{2} x}{\mathrm{~d} t^{2}}+\frac{c}{m} \frac{\mathrm{d} x}{\mathrm{~d} t}+\frac{k}{m} x=\frac{1}{m} F(t)
$$

where, $m$ is the mass of the aircraft, $c$ is the damping coefficient, and $k$ is the spring constant. $F(t)$ is the force of the moment. In this study, the forcing function is assumed to be the free motion. Let us assume $x=A e^{\lambda t}$ and free motion $(F(t)=0)$. Then, Eq. (9) can be written as follows:

$$
\lambda^{2}+\frac{c}{m} \lambda+\frac{k}{m}=0
$$

Here, $\lambda$ represents the eigenvalues in Eq. (9), which is called the characteristic equation. The $\lambda$ values of the characteristic equation are called the characteristic roots and eigenvalues of the flight motion. In this study, the longitudinal motion is considered, and $\lambda$ is used to predict the specific mode of this longitudinal motion such as the phugoid or short-period mode. The $\lambda$ values of Eq. (9) are found using Eq. (10):

$$
\lambda_{1,2}=-\frac{c}{2 m} \pm \sqrt{\left(\frac{c}{2 m}\right)^{2}-\frac{k}{m}}
$$

In this study, the target condition for the stable mode of longitudinal motion can be estimated using the real and imaginary roots of $\lambda$, as shown in Fig. 2 . The damping can be estimated by $c=\zeta c_{c r}$ and $c_{c r}=2 \sqrt{\mathrm{km}}$. Here, the $\zeta$ term is the damping ratio if $\zeta=0, c=0$ and the mode is an un-damped oscillation. Then, the natural frequency, called the un-damped natural frequency, can be written as $\omega_{n}=\sqrt{\frac{k}{m}}$. By substituting the terms for the damping ratio $\zeta$ and natural frequency $\omega_{n}$ for terms in Eq. (9), the following equation can be expressed [2]:

$$
\lambda^{2}+2 \zeta \omega_{n} \lambda+\omega_{n}{ }^{2}=0
$$

From Eq. (11), the characteristic roots are expressed as Eq. (12):

$$
\lambda_{1,2}=-\zeta \omega_{n} \pm i \omega_{n} \sqrt{1-\zeta^{2}}
$$

In this study, the SDM's center of gravity is moving along a longitudinal and horizontal line at a constant velocity, and the pitching motion is self-excitation. The governing equation for the pure pitching motion can be written as Eq. (13):

$$
\sum M_{c g}=I_{y y} \ddot{\theta}
$$

Here, $M_{c g}$ is the pitching moment, $I_{y y}$ is the moment inertia in the $y$ direction, and $\ddot{\theta}$ is the pitch angle time rate. The variables are the angle of attack $\alpha$, pitch angle $\theta$, and time rates of change for these variables $\alpha$ and $\dot{\theta}$. The following equation can be used to evaluate the moment:

$$
\Delta M=f(\Delta \alpha, \Delta \dot{\alpha}, \Delta q)
$$

Eq. (15) can be expanded in terms of the perturbation variables by means of the Taylor series form:

$$
\Delta M=\frac{\partial M}{\partial \alpha} \Delta \alpha+\frac{\partial M}{\partial \dot{\alpha}} \Delta \dot{\alpha}+\frac{\partial M}{\partial q} \Delta q
$$

By aligning the body and fixed coordinate at $t=0$, the assumption of change in angle of attack and pitch angles could be identified as equal. Eq. (16) can be replaced by this identified equal with Eq. (15) [2].

$$
\Delta \alpha=\Delta \theta \text { and } \Delta \dot{\theta}=\Delta q=\Delta \dot{\alpha}
$$




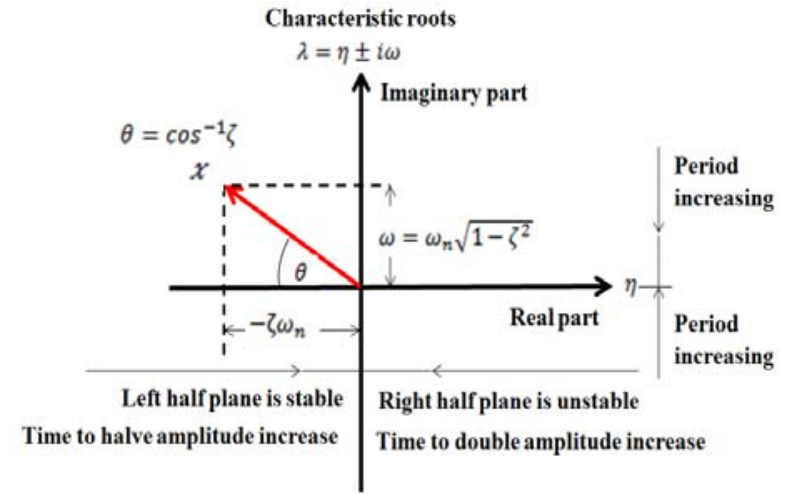

Fig. 2 Characteristics roots of flight simulation analysis [2].

Substituting Eq. (16) into Eq. (15) yields the following Eq. (17):

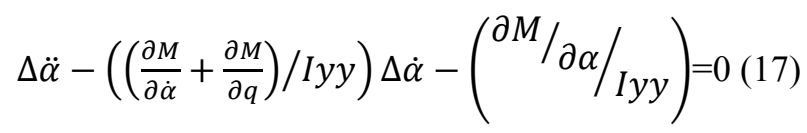

Eq. (17) can be rearranged by means of Eq. (18). Therefore, the equation of motion for the pure pitching motion can be obtained as Eq. (18):

$$
I_{y y} \ddot{\theta}-\left(\frac{\partial M}{\partial \dot{\alpha}}+\frac{\partial M}{\partial q}\right) \dot{\theta}-\frac{\partial M}{\partial \alpha} \theta=0
$$

Based on Eq. (18), the arrangement used for Eq. (9) can be used as follows:

$$
\lambda^{2}-\left(M_{q}+M_{\dot{\alpha}}\right) \lambda-M_{\alpha}=0
$$

Eq. (19) can be compared with the standard equation of motion such as Eq. (11).

In the flight simulation analysis, it is better to use a non-dimensional form for the prediction. Using Eq. (20) with the aerodynamic force, moment and derivative coefficient, the longitudinal motion of the aerodynamic coefficient can be written as Eq. (20):

$$
\begin{gathered}
C_{i}=C_{i 0}(\alpha, \beta, M)+\bar{C}_{i q}(\alpha, M, q) \cdot \frac{c q}{2 U_{\infty}}+ \\
C_{i \delta}(\alpha, M, \delta) \cdot \delta
\end{gathered}
$$

where,

$$
\bar{C}_{i q}=C_{i_{q}}+C_{i_{\dot{\alpha}}}
$$

and

$i=X, Z-$ force \& Pitching moment coefficients.

From Eq. (20), the prediction requires estimations of the longitudinal stability coefficients for the $X$-force derivatives such as $C_{x u}$ and $C_{x a}$. Table 1 lists the symbols and meanings of the longitudinal stability coefficients. In addition, for the $Z$-force derivatives, stability coefficients such $C_{Z q}, C_{Z \dot{\alpha}}$ and $C_{Z \alpha}$ are involved in the calculation of this axis. $C_{m q}, C_{m \dot{\alpha}}$ and $C_{m a}$ are used for the pitching moment derivatives. In this study, $C_{X \dot{\alpha}}$ and $C_{X q}$ are basically set to zero because they are very small values. $C_{m u}$ is assumed to be zero because of the low speed condition, whereas $C_{Z u}$ is directly calculated from the value of $C_{L(\alpha=0)}$, based on the wing characteristics of the SDM. All the longitudinal stability coefficients are expected to be obtained by using the USAF Stability and Control DATCOM at the initial stage of the prediction method.

3.3.2 State Variable by Means of Equations of Motion

In the mathematical form of the differential equation, the state space equation is usually used in matrix form to make the calculation easier. Eq. (21) shows a representation of the state space equation.

$$
\dot{x}=A x
$$

where, $x$ represents the state variables, and matrix $A$ contains the SDM's dimensional stability derivatives. In this study, the state variables include the axial velocity $u$, normal velocity $w$, pitch rate $q$ and pitch angle $\theta$. By rearranging Eq. (21), the full matrix form is produced, as shown in Eq. (22).

$$
\begin{gathered}
{\left[\begin{array}{c}
\Delta \dot{u} \\
\Delta \dot{w} \\
\Delta \dot{q} \\
\Delta \dot{\theta}
\end{array}\right]} \\
=\left[\begin{array}{ccc}
X_{u} & X_{w} & 0-g \\
Z_{u} & Z_{w} & u_{0} 0 \\
M_{u}+M_{\dot{w}} Z_{u} & M_{w}+M_{\dot{w}} Z_{u} & M_{q}+M_{\dot{w}} u_{0} \\
0 & 0 & 10
\end{array}\right] \\
{\left[\begin{array}{c}
\Delta u \\
\Delta w \\
\Delta q \\
\Delta \theta
\end{array}\right]}
\end{gathered}
$$

The SDM's dimensional stability derivatives for matrix $A$ are obtained from the $X$ - and $Z$-force derivatives and pitching moment derivatives, as listed in Table 2 for simulation 1, Table 4 for simulation 2, and Table 6 for simulation 3 . 
Table 1 Longitudinal stability coefficients meaning and symbols.

\begin{tabular}{ll}
\hline Longitudinal stability coefficients & Symbol \\
\hline$X, Z$-force and pitch moment curve slope coefficient & $C_{X \alpha}, C_{Z \alpha}, C_{m \alpha}$ \\
$Z$-force and pitch moment coefficient due to time rate of change of angle of attack & $C_{Z \dot{\alpha}}, C_{m \dot{\alpha}}$ \\
$Z$-force and pitch moment coefficient due to pitching velocity & $C_{Z q}, C_{m q}$ \\
$X$-force coefficient due to change of forward speed & $C_{X u}$ \\
\hline
\end{tabular}

Table 2 Dimensional stability derivatives matrix $A$-simulation 1.

\begin{tabular}{lllll}
\hline State variables & \multicolumn{2}{l}{ Dimensional stability derivatives, $A$} & -9.81 \\
\hline$\Delta \dot{\mathbf{u}}$ & -0.04692022 & 8.577349 & 0 & 0 \\
$\Delta \dot{\mathbf{w}}$ & -4.985018511 & -7.15668 & 301.9406 & 0 \\
$\Delta \dot{\mathbf{q}}$ & 0.391890503 & -2.44893 & -23.8264 & 0 \\
$\Delta \dot{\boldsymbol{\theta}}$ & 0 & 0 & 1 & 0 \\
\hline
\end{tabular}

Table 3 Characteristics roots for long period and short period motion—simulation 1.

\begin{tabular}{ll}
\hline Exact method & Approximation method \\
\hline (Phugoid) $=\lambda_{1,2}=-0.0239 \pm \mathrm{i} 0.3925$ & (Phugoid) $=\lambda_{1,2}=-0.0234 \pm \mathrm{i} 0.4013$ \\
(Short period) $=\lambda_{3,4}=-15.4911 \pm \mathrm{i} 26.6936$ & (Short period) $=\lambda_{3,4}=-15.4915 \pm \mathrm{i} 25.8842$ \\
\hline
\end{tabular}

Table 4 Dimensional stability derivatives matrix $A$-simulation 2.

\begin{tabular}{lllll}
\hline State variables & \multicolumn{2}{l}{ Dimensional stability derivatives, $A$} & \\
\hline$\Delta \dot{\mathbf{u}}$ & -0.02548787 & 6.110081 & 0 & -9.81 \\
$\Delta \dot{\mathbf{w}}$ & -4.719879237 & -7.62745 & 308.2892196 & 0 \\
$\Delta \dot{\mathbf{q}}$ & 0.052303228 & -44.8581 & -22.03096175 & 0 \\
$\Delta \dot{\boldsymbol{\theta}}$ & 0 & 0 & 1 & 0 \\
\hline
\end{tabular}

Table 5 Characteristics roots for long period and short period motion-simulation 2.

\begin{tabular}{ll}
\hline Exact method & Approximation method \\
\hline (Phugoid) $=\lambda_{1,2}-0.03 \pm \mathrm{i} 0.38$ & (Phugoid) $=\lambda_{1,2}-0.007654 \pm \mathrm{i} 0.387$ \\
(Short period) $=\lambda_{3,4}=-14.81 \pm \mathrm{i} 117.4$ & (Short period) $=\lambda_{3,4}=-14.82 \pm \mathrm{i} 117.38$ \\
\hline
\end{tabular}

Table 6 Dimensional stability derivatives matrix $A$-simulation 3.

\begin{tabular}{lllll}
\hline State variables & \multicolumn{1}{l}{ Dimensional stability derivatives, $A$} & \\
\hline$\Delta \dot{\mathbf{u}}$ & -0.025070263 & 8.115254 & 0 & -9.81 \\
$\Delta \dot{\mathbf{w}}$ & -4.51358648 & 7.494124 & 303.2380364 & 0 \\
$\Delta \dot{\mathbf{q}}$ & 0.068357101 & -46.286 & -25.95374242 & 0 \\
$\Delta \dot{\boldsymbol{\theta}}$ & 0 & 0 & 1 & 0 \\
\hline
\end{tabular}

In general, Fig. 3 shows the procedure for evaluating the flight simulation in this study.

\section{Computational Condition of Present Study}

In this study, three simulation cases are considered. Then, the results are compared with the results of a simulation without the Kriging model. LHS (Latin hypercube sampling) is applied to select the sample points. In the first simulation, five samples are used. In the second simulation, 20 samples are used, and in the third simulation, 50 samples are used.

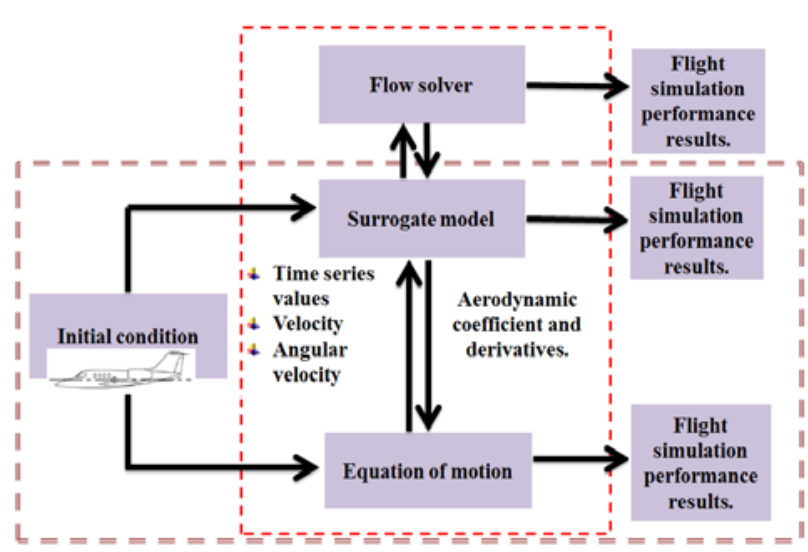

Fig. 3 Procedure for flight simulation analysis. 


\section{Results and Discussions}

\subsection{Prediction of Aerodynamic Coefficient/Derivatives by Kriging Model}

\subsubsection{Comparison between Predicted and Exact} Results

To investigate the Kriging model for predictions, a comparison is made between the predicted and exact values (DATCOM). Figs. 4-6 show example graphs comparing the exact functions and predicted functions using Kriging models constructed with 20 sample points for stability coefficients $C_{X u}$ and $C_{X \alpha}$, five samples points for $C_{Z a}$, and 50 sample points for $C_{Z \dot{a}}, C_{Z q}, C_{m a}, C_{m \dot{a}}$ and $C_{m q}$, respectively. In Figs. $4 \mathrm{a}$ and $4 \mathrm{~b}$, the results of the Kriging function are similar to those of the exact function for the $X$-force functions. On the other hand, Fig. 5 shows comparisons for the $Z$-force functions, $C_{Z a}, C_{Z \dot{a}}$ and $C_{Z q}$, respectively. Fig. 6 shows comparisons for the pitching moment functions $C_{m a}, C_{m \dot{a}}$ and $C_{m q}$, respectively. Different sample points are used in this study to determine the dependency on the number of samples. Generally speaking, a larger number of sample points will result in greater agreement between the predicted function and the exact function in this study.

\subsubsection{Trend of Coefficient and Derivatives}

In this study, the prediction process is difficult because some coefficients can be fit with linear functions, whereas others are multi-modal data when a large number of sample points are involved. The aerodynamic coefficients and derivatives obtained by using the Kriging model are used in Eq. (22). The predicted function of the Kriging model can be viewed as a contour plot to observe how the trend pattern of the aerodynamic model is affected by the two variables used in this study: the Mach number and angle of attack. Fig. 7a shows an example of axial force coefficient $C X$ for the angle of attack and Mach number, Fig. $7 \mathrm{~b}$ shows coefficient $C Z$ and Fig. 7c shows the contour for the pitching moment coefficient $\mathrm{Cm}$. All the contours were plotted by choosing the 50 sample points as examples.

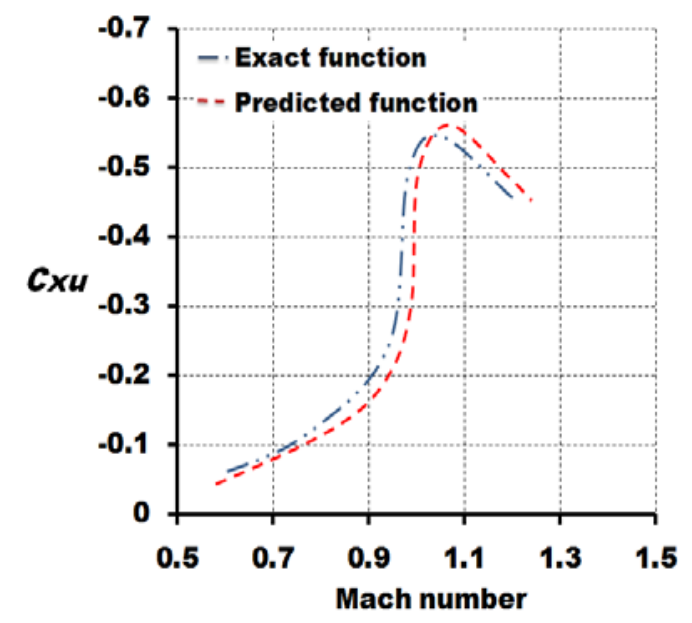

(a)

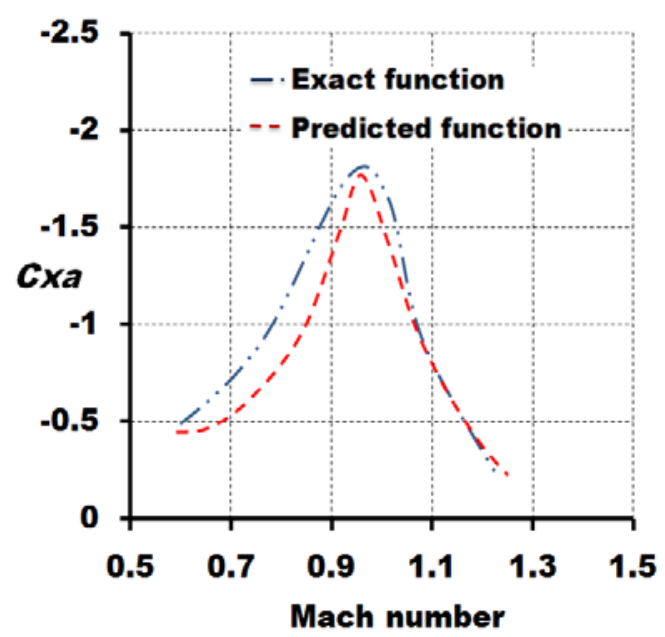

(b)

Fig. 4 Comparisons between exact function and predicted function for $X$-force derivative coefficients due to: (a) forward speed and (b) angle of attack at $0^{\circ}$.

According to Fig. 7a, the minimum $C X$ could be obtained at Mach number 1.0 and an angle of attack of $10.0^{\circ}$. On the other hand, Fig. $7 \mathrm{~b}$ shows the highest $C Z$. This is a reasonable result because of the Mach number divergence situation. According to Fig. $7 \mathrm{c}$ at an angle of attack of $25.0^{\circ}$, the minimum pitching moment coefficient $\mathrm{Cm}$ is expected to occur at a Mach number range of 0.88-1.30. The pitching moment coefficient $\mathrm{Cm}$ must also have a suitable value to evaluate the damping and frequency [2].

\subsection{Application to Longitudinal Motion}

In an application to determine the longitudinal motion, Eq. (22) is solved, using the aerodynamic 

and Derivatives by Surrogate Model Approach

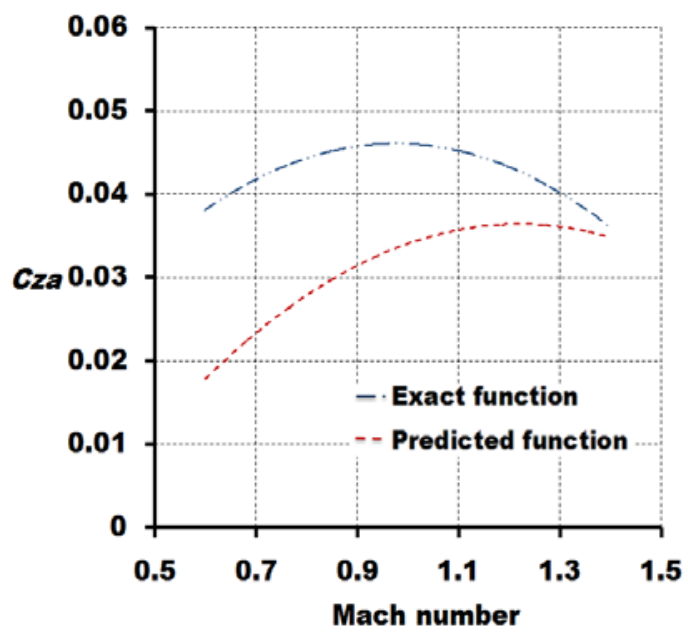

(a)

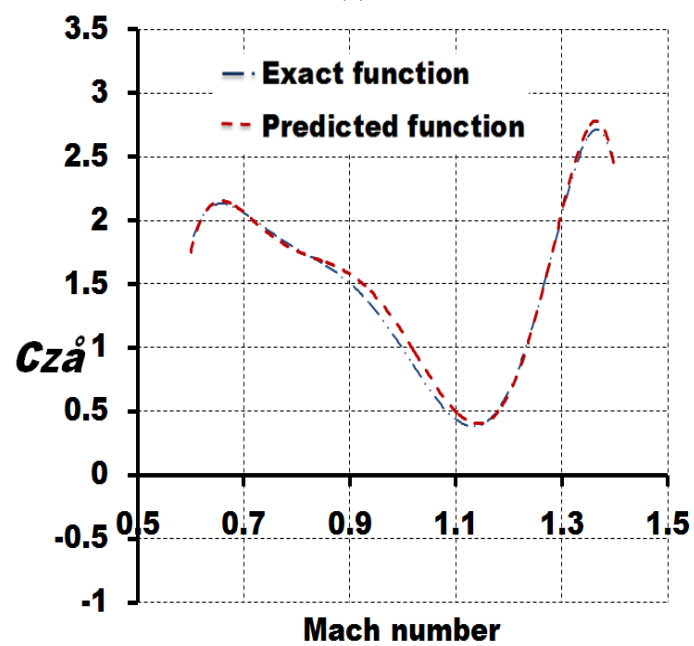

(b)

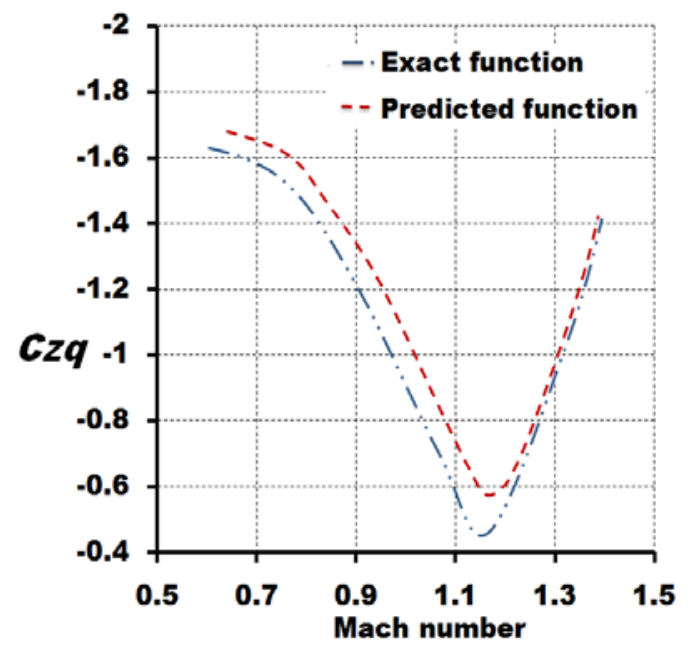

(c)

Fig. 5 Comparisons between exact function and predicted function for $Z$-force derivative coefficients due to: (a) angle of attack at $0^{\circ}$, (b) angle of attack derivatives and (c) pitching velocity.

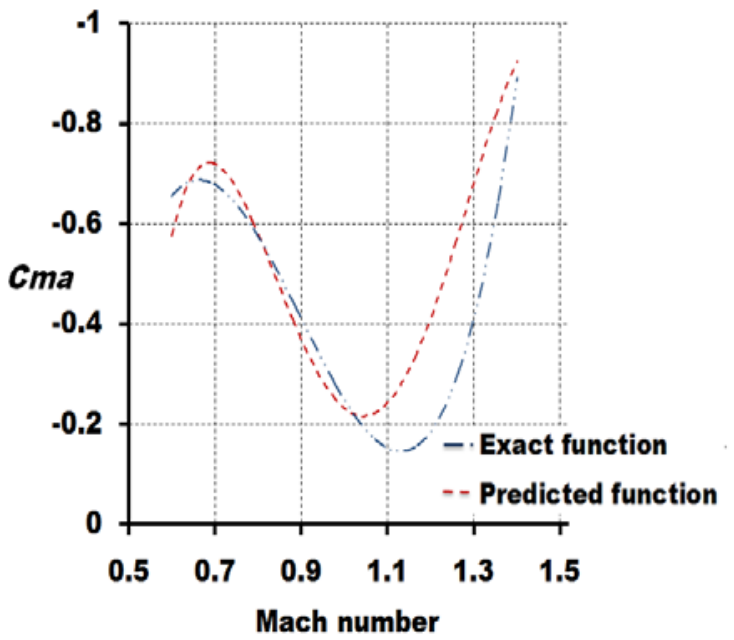

(a)

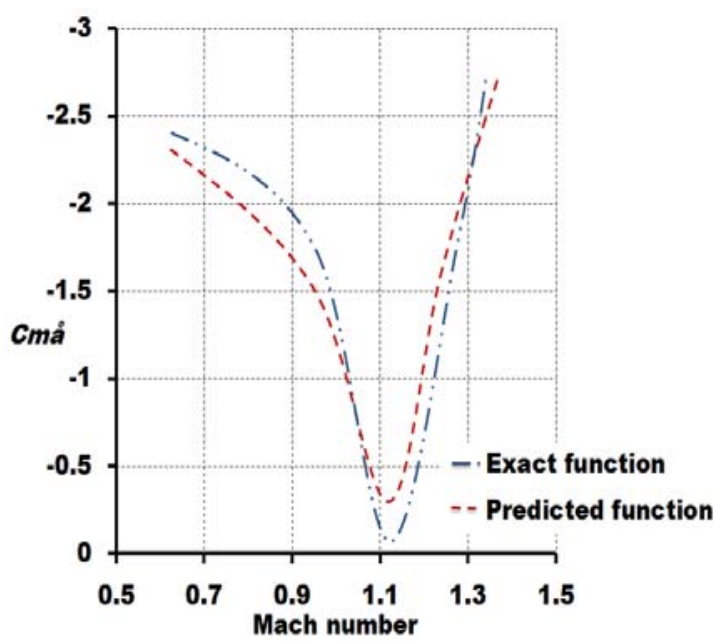

(b)

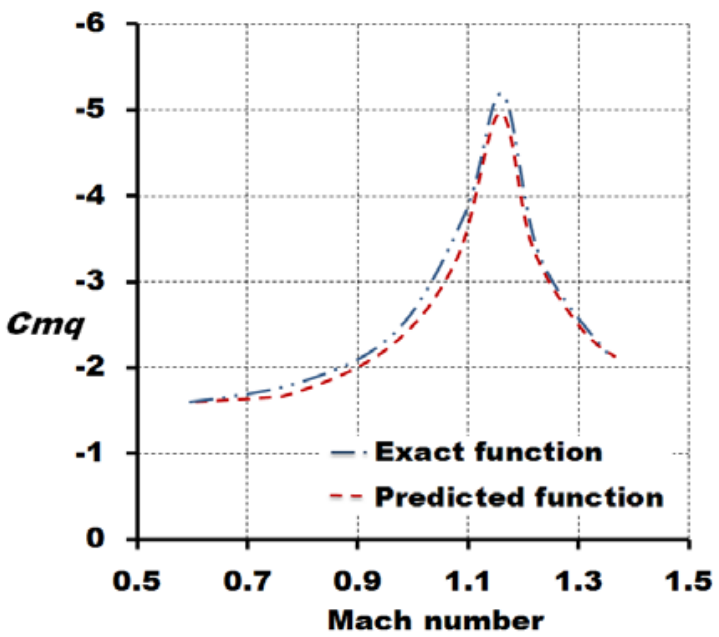

(c)

Fig. 6 Comparisons between exact function and predicted function for pitching moment derivative coefficients due to: (a) angle of attack at $0^{\circ}$, (b) angle of attack derivatives and (c) pitching velocity. 


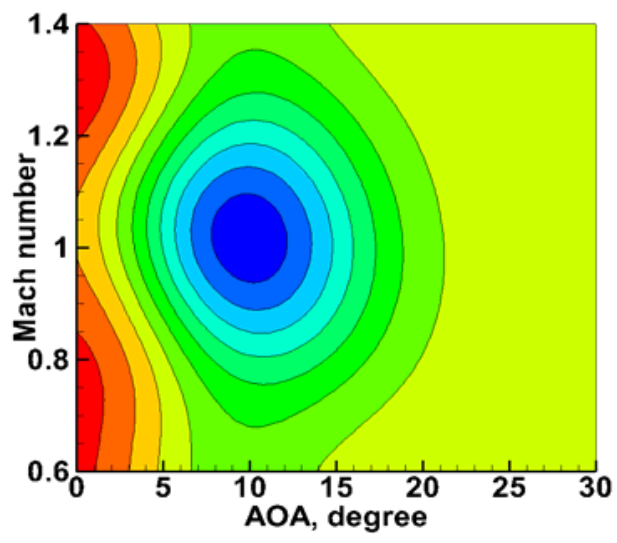

(a)

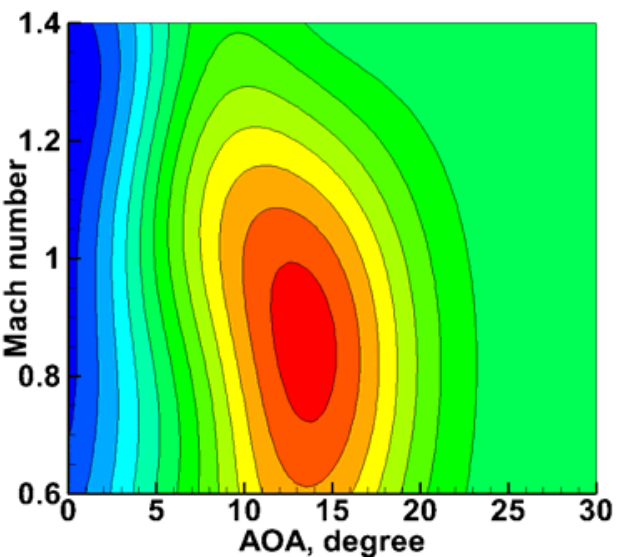

(b)

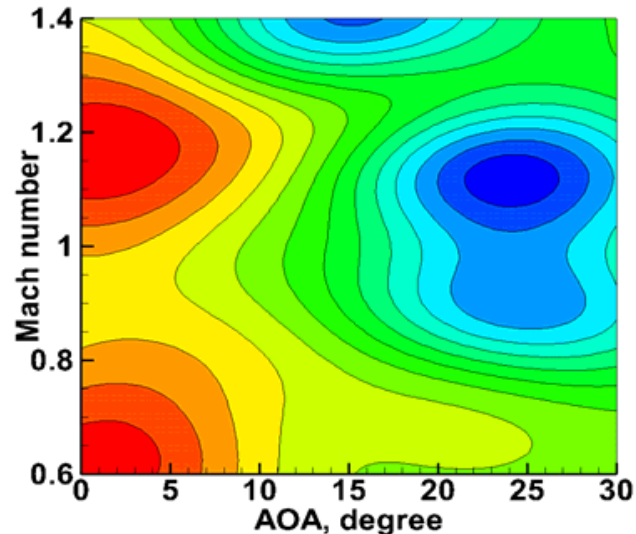

(c)

Fig. 7 Functions predicted by Kriging model: (a) axial force coefficient CA contour, (b) normal force $C Z$ coefficient contour and (c) pitching moment coefficient $\mathrm{Cm}$ contour.

coefficients and derivatives for the longitudinal direction. After this, the value of $\lambda$ will be obtained and used to describe the two types of longitudinal modes: the phugoid (long period) and short-period modes. The characteristic $\lambda$ values for three simulation samples are listed in Tables 3, 5 and 7, including the exact solution and Kriging model.

The easiest way to examine the flight simulation is based on the determination of the damping $\zeta$ and natural frequency $w_{n}$ based on the equation of motion. In addition, others motion parameters are also listed in Tables 8 and 9 , including the time to the half mode $t_{1 / 2}$, period of the mode, and number of cycles. These characteristics include error if the accuracy of the prediction databases is lower than that of the exact databases. Using these motions, the phugoid (long period) mode and short-period mode are simulated. The error comparison results for the phugoid motion are listed in Table 8, whereas those for the short period are listed in Table 9.

In the case of the phugoid mode (Table 8), simulations 2 and 3 show the closest results for all the characteristic motions. However, only the period and damped frequency show close results for the exact and Kriging model predictions. Thus, the period and damped frequency are considered to be the main criteria for evaluating the performance of the phugoid mode. Simulation 1 also has small values for the period, damping $\zeta$ and natural frequency $w_{n}$. The phugoid mode time series is shown in Fig. 9. According to Fig. 9, simulation 3 is very suitable with the largest damping ration and time period. In the flight simulation analysis, the phugoid mode occurs very slowly, which allows the pilot to control and correct the phugoid mode during an extremely dangerous flight if the damping becomes too low. Therefore, to maintain sufficient stability, the design must ensure that the damping ratio is never too low. Thus, simulation 3 has the largest damping ratio compared to the other simulations, as shown in Fig. 9. The simulation with the largest damping ratio is considered to be the best in this study.

In contrast, for the short-period mode (Table 9), the results of the characteristic motion for simulations 1,2 and 3 show good agreement. In predicting the short-period mode, critical judgment is more important 
Table 7 Characteristics roots for long period and short period motion—simulation 3.

\begin{tabular}{ll}
\hline Exact method & Approximation method \\
\hline (Phugoid) $=\lambda_{1,2}=-0.04 \pm \mathrm{i} 0.38$ & (Phugoid) $=\lambda_{1,2}=-0.0125 \pm \mathrm{i} 0.382$ \\
$($ Short period $)=\lambda_{3,4}=-16.70 \pm \mathrm{i} 118.26$ & (Short period) $=\lambda_{3,4}=-4.6291 \pm \mathrm{i} 119$ \\
\hline
\end{tabular}

Table 8 Long period characteristic comparison between exact and approximated values.

\begin{tabular}{|c|c|c|c|c|c|c|c|c|c|}
\hline \multirow[b]{2}{*}{ Parameters } & \multicolumn{3}{|c|}{ Exact } & \multicolumn{3}{|c|}{ Approximation } & \multicolumn{3}{|c|}{ Error(\%) } \\
\hline & Sim. 1 & Sim. 2 & Sim. 3 & Sim. 1 & $\operatorname{Sim} .2$ & Sim. 3 & Sim. 1 & Sim. 2 & Sim. 3 \\
\hline Time to half, $t_{1 / 2}(\mathrm{~s})$ & 28.87 & 23 & 17.25 & 29.487 & 54.143 & 55.5 & 2.09245 & 57.5199 & 68.91891892 \\
\hline Period (s) & 16.008 & 16.534 & 16.53 & 15.657 & 16.235 & 16.45 & 2.241809 & 1.8417 & 0.486322188 \\
\hline $\mathrm{N}$-cycles $\left(\mathrm{N}_{1 / 2}\right)$ & 1.806 & 1.393 & 1.045 & 1.886 & 3.35 & 3.36 & 4.24178 & 58.4179 & 68.89880952 \\
\hline Damping $(\zeta)$ & 0.0608 & 0.0787 & 0.1046 & 0.0583 & 0.032887 & 0.132814 & 4.288165 & 139.3043 & 18.7663802 \\
\hline Damped frequency $\left(\omega_{d}\right)$ & 0.293 & 0.3811 & 0.382 & 0.202 & 0.3875 & 0.388 & 2.23881 & 1.65161 & 0.000000 \\
\hline
\end{tabular}

Table 9 Short-period characteristic comparison between exact and approximated values.

\begin{tabular}{|c|c|c|c|c|c|c|c|c|c|}
\hline \multirow[b]{2}{*}{ Parameters } & \multicolumn{3}{|c|}{ Exact } & \multicolumn{3}{|c|}{ Approximation } & \multicolumn{3}{|c|}{ Error $(\%)$} \\
\hline & Sim. 1 & Sim. 2 & Sim. 3 & Sim. 1 & Sim. 2 & Sim. 3 & Sim. 1 & Sim. 2 & Sim. 3 \\
\hline Time to half, $t_{1 / 2}(\mathrm{~s})$ & 0.0445 & 0.04659 & 0.0413 & 0.0445 & 0.04653 & 0.0413 & 0.00000 & 0.12895 & 0.00000 \\
\hline Period (s) & 0.2354 & 0.05347 & 0.053 & 0.2427 & 0.0535 & 0.0533 & 3.007829 & 0.056075 & 0.0562852 \\
\hline $\mathrm{N}$-cycles $\left(\mathrm{N}_{1 / 2}\right)$ & 0.1895 & 0.8727 & 0.779 & 0.1838 & 0.8707 & 0.775 & 3.1012 & 0.2297 & 0.51613 \\
\hline Damping $(\zeta)$ & 0.5019 & 0.125 & 0.1398 & 0.5135 & 0.125 & 0.1405 & 2.259007 & 0.00000 & 0.498221 \\
\hline Damped frequency $\left(\omega_{\boldsymbol{d}}\right)$ & 30.86 & 118.48 & 119.43 & 30.165 & 118.31 & 119.00 & 2.30399 & 0.14369 & 0.36134 \\
\hline
\end{tabular}

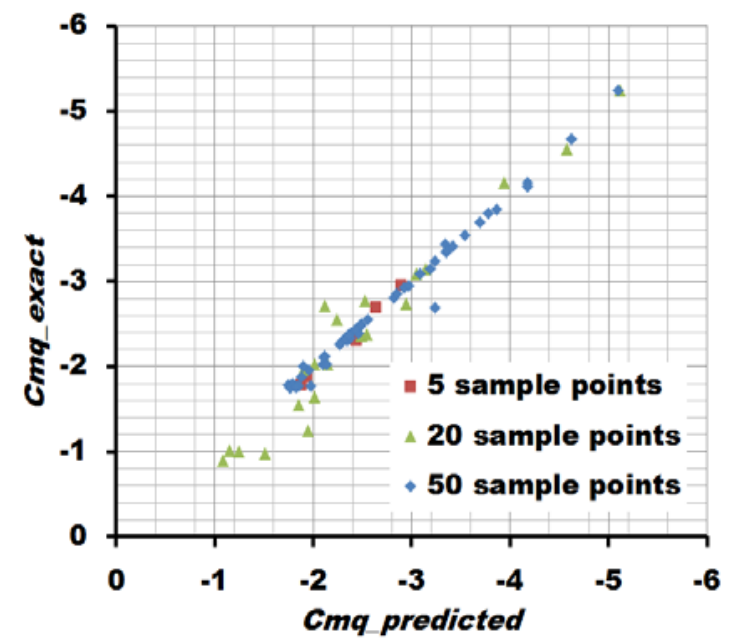

Fig. 8 Comparisons of numbers of sample points for $C_{m q}$ exact function versus predicted function.

than the phugoid mode. If this short-period mode has a high frequency and is heavily damped, the aircraft will respond rapidly [2]. In relation to the characteristic mode, sim. 3 has a small error reading because the values of all the characteristic modes for sims. 1 and 2 are close to those for sim. 3. In relation to the characteristic mode of motion, the time series plot of the short-period mode is shown in Fig. 10. According to Fig. 10, sim. 3 has a short-period mode under the condition of an under-damped exponentially decaying motion, just as with the phugoid motion. Because of the small error in sim. 3, it is a reasonable simulation for the prediction of the short-period mode based on aerodynamic coefficients and derivatives, and has good agreement with the exact solution.

The dependency on the number of sample points is investigated. Fig. 8 shows an example of $C_{m q}$. It is one of the aerodynamic derivatives that are significant when choosing the number of sample points in this study. According to Fig. 8, the cases with 5 and 50 sample points show little error between the exact and predicted results. For the phugoid mode, $C_{m q}$ is more critical usage than in the short-period mode. For the phugoid mode, the case with five sample points shows less error than those with 20 and 50 sample points (Table 8), However, because of Eq. (22), the overall results for the phugoid mode shows that 50 sample points produces better results than the other numbers 


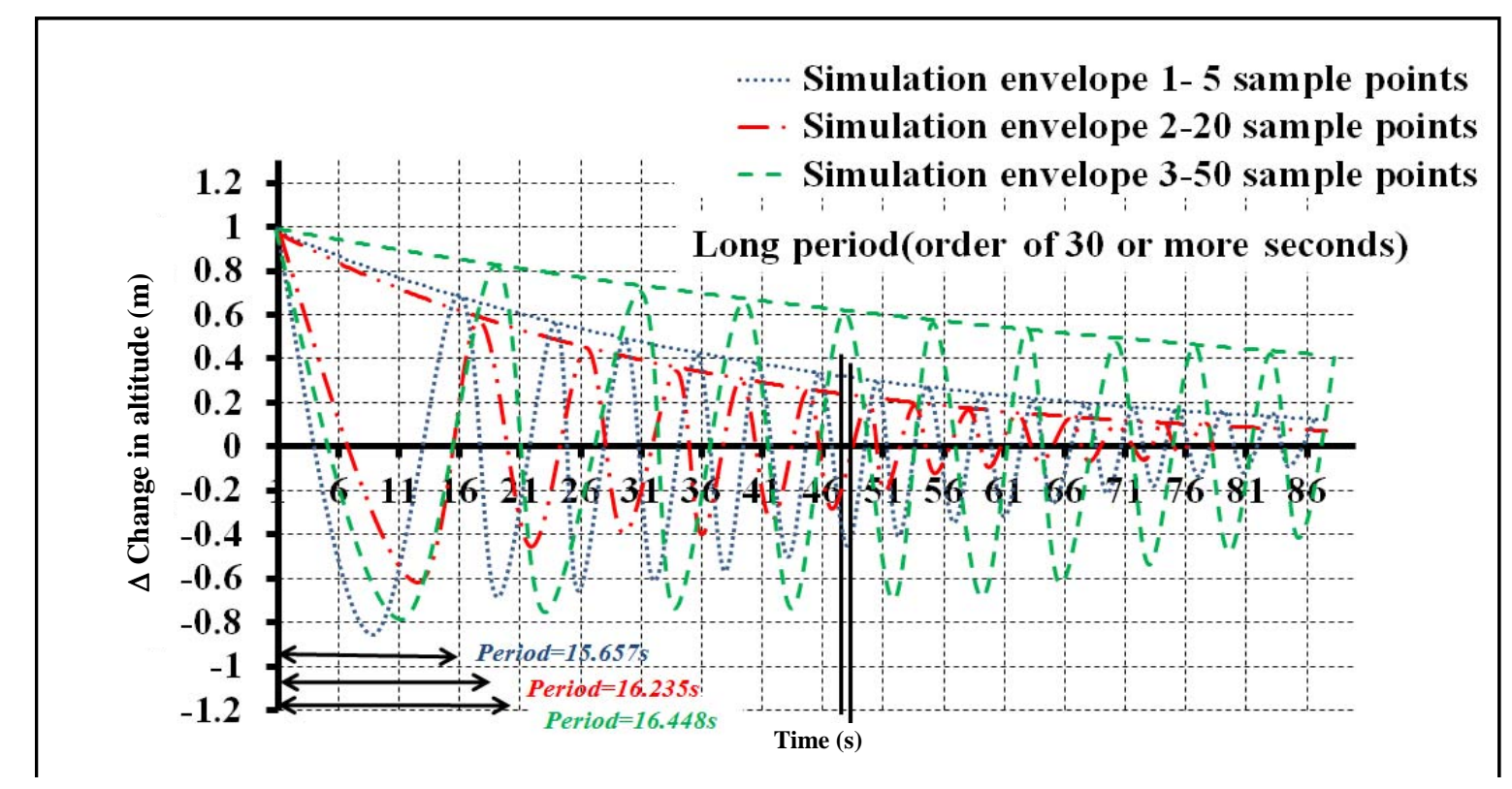

Fig. 9 Time series plot of phugoid mode for sims. 1, 2 and 3.

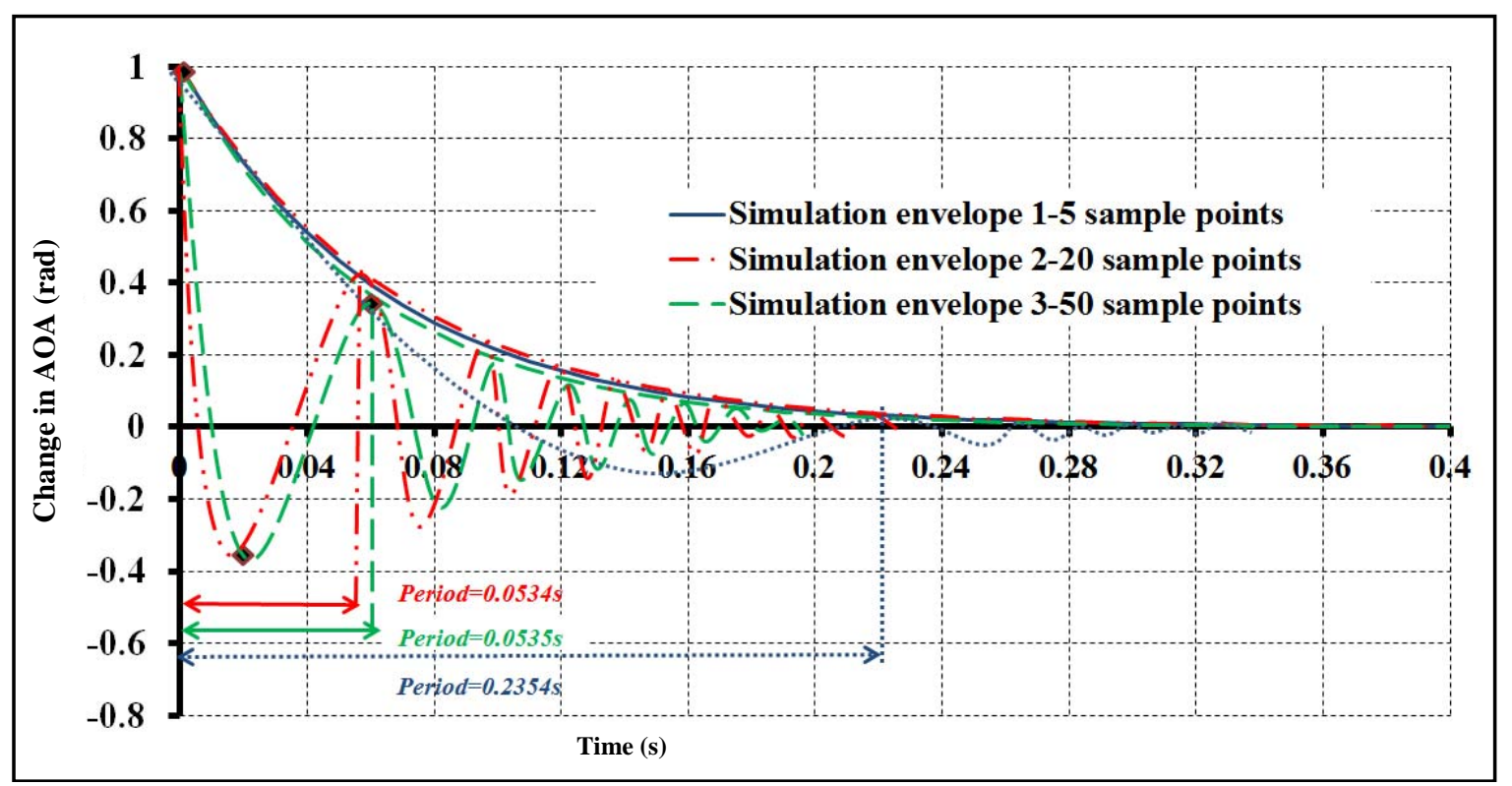

Fig. 10 Time series plot of short-period mode for sims. 1, 2 and 3.

of sample points. It proves that for phugoid more the greater number of sample points, the better the phugoid mode. In contrast, for the short-period mode, $C_{m q}$, is not the main aerodynamic derivatives to effect the short-period mode, the main derivatives are from $X$ - and $Z$-force. Thus, the small error between the cases with 5 and 50 sample points does not affect the overall performance of the short-period mode (Eq. (22)). Therefore, the results for the short period still show good agreement among the sample points. An accurate database is important for the prediction process. Moreover, the results of a comparison of sims. 1,2 and 3 are listed for both the phugoid and short-period modes in Tables 8 and 9, respectively.

\section{Conclusions}

In conclusion, this paper detailed the surrogate model development and construction process from the 
initial stage using the USAF Stability and Control DATCOM. In addition, accurate databases based on Kriging models showed good agreement in flight simulation prediction through time series plots, with a larger number of sample points producing better approximation results. In addition, the prediction process of longitudinal motion based aerodynamic coefficients and derivatives are begun from an equation of motion. Lastly, the prediction method using the Kriging model was verified in comparison to the exact function. Good agreement was found for short-period motion, with an average error of $0 \%-3 \%$ for the criteria of several characteristic motions. Based on the characteristic motions for the phugoid and short-period modes, sim. 3 was considered to be the best predicted motion based on the predicted aerodynamics coefficients and derivatives. However, for the phugoid mode, the approximation did not show good agreement between the two methods and large errors occurred. In a future study, the use of the established method for lateral motion will be tested.

\section{Acknowledgments}

The author would like to wish for special thanks for stud fund by the AHRF (Asian Human Resources Fund) from Tokyo Metropolitan Government to the TMU (Tokyo Metropolitan University). In addition, many thanks go to my supervisor for his guidance and support. Lastly, all members in Kanazaki laboratory TMU, thank you about their share knowledge, teaching, and advice in the course of completing this paper.

\section{References}

[1] USAF Stability and Control DATCOM, Flight Control Division, Air Force Flight Dynamics Laboratory, Wright-Patterson Air Force Base, Fairborn, OH.

[2] Nelson, R. C. 1998. Flight Stability and Automatic Control. 2nd Edition. New York: McGraw-Hill.
[3] Jeong, S., Murayama, M., and Yamamoto, K. 2005. "Efficient Optimization Design Method Using Kriging Model." Journal of Aircraft 42 (2): 413-20.

[4] Donald, R. J. 2001. "A Taxonomy of Global Optimization Methods Based on Response Surfaces." Journal of Global Optimization 21: 345-83.

[5] Shahrokhi, A., and Jahangirian, A. 2007. "An Efficient Aerodynamics Optimization Method Using a Genetic Algorithm and a Surrogate Model." Presented at the 16th Australasian Fluid Mechanics Conference, Austria.

[6] Ueno, M., and Miwa, H. 2001. "New Dynamics Stability Equipment for Transonic Wind Tunnel Testing at NAL." Presented at the 39th AIAA Aerospace Sciences Meeting and Exhibit, Nevada.

[7] Narita, Y., Hashimoto, A., and Kanazaki, M. 2012. "Numerical Simulation: Flight Dynamics Stability Analysis Using Unstructured Based Navier-Stokes Solver." Presented at the Asia-Pacific International Symposium on Aerospace Technology, Korea.

[8] Schmidt, E. 1985. Standard Dynamics Model Experiments with the DFVLR/AVA Transonic Derivative Balance. Technical report for National Technical Information Service, USA.

[9] Hashimoto, A., Hashizume, M., Sunada, S., Ueno M., and Murakami, K. 2013. "Unsteady Analysis of Aerodynamic Derivatives on Standard Dynamics Model." Presented at the 51st AIAA Aerospace Sciences Meeting including the New Horizons Forum and Aerospace Exposition, Texas.

[10] Kaenel, R. V., Rizzi, A., Oppelstrup, J., Grabowski, T. G., Ghoreyshi, M., and Cavagna, L. 2008. "CEASIOM: Simulating Stability and Control with CFD/CSM in Aircraft Conceptual Design." Presented at 26th International Congress of the Aeronautical Sciences, Alaska, USA.

[11] Badufle, C., Blondel, C., and Druot, T. 2010. "A Heuristic-Based Framework to Solve a Complex Aircraft Sizing Problem." Journal of Engineering Applications of Artificial Intelligence 23: 704-14.

[12] Kanazaki, M., Tanaka, K., Jeong, S., and Yamamoto, K. 2006. "Design Exploration of High-Lift Airfoil Using Kriging Model and Data Mining Technique.” Presented at the European Conference on Computational Fluid Dynamics, the Netherlands.

[13] Lee, K. H., and Park, F. J. 2006. "A Global Robust Optimization Using Kriging Based Approximation Model." JSME International Journal Series C 49 (3): 779-88. 